\title{
Sigma-terms in heavy baryon chiral perturbation theory revisited
}

\author{
B. Borasoy中 \\ Department of Physics and Astronomy \\ University of Massachusetts \\ Amherst, MA 01003, USA
}

\begin{abstract}
The $\sigma$-terms are calculated at next-to-leading order in heavy baryon chiral perturbation theory by employing a cutoff regularization. The results do not depend on the cutoff value to the order we are working. The baryon masses and $\sigma_{\pi N}(0)$ are used to perform a least-squares fit to the three appearing low-energy constants and predictions for the two $K N \sigma$-terms and the strange contribution to the nucleon mass are made. The lack of convergence in the chiral expansions of these quantities when regularized dimensionally is overcome in the cutoff scheme. The $\sigma$-term shifts to the pertinent Cheng-Dashen points are calculated. We also include the spin- $3 / 2$ decuplet in the effective theory.
\end{abstract}

\footnotetext{
${ }^{1}$ email: borasoy@het.phast.umass.edu
} 


\section{Introduction}

Chiral perturbation theory which is the effective field theory of the Standard Model at low energies in the hadronic sector has been successfully applied within the sector of Goldstone Bosons [1]. However, traditional SU(3) heavy baryon chiral perturbation theory does not appear to work well. The leading nonanalytic components from loop corrections destroy the good experimental agreement which exists at lowest order. The additional contributions have to be compensated by higher order countertems. This leads to problems with the convergence of the chiral series.

Recently a resolution of this problem was proposed by using a cutoff regularization instead of the common dimensional regularization scheme [2, 3]. There, the authors come to the conclusion that dimensionally regularized Feynman diagrams carry implicit and large contributions from short distance physics. In contrast, the cutoff scheme picks out the long distance part of the integral, which behaves as expected on physical grounds. Both an exponential cutoff in threemomentum and a dipole regulator were employed therein. However, in the cases discussed there the cutoff is irrelevant - a consistent chiral expansion can then be carried out.

In these works an analysis of the octet baryon masses has been given using the lowest and next-to-leading order in the derivative expansion of the effective Lagrangian. Further information on the scalar sector of baryon CHPT is given by the scalar form factors or $\sigma$-terms which measure the strength of the various matrix elements $m_{q} \bar{q} q(q=u, d, s)$ in the proton and vanish in the chiral limit of zero quark masses. Thus, they are particularly suited to test our understanding of spontaneous and explicit chiral symmetry breaking. The purpose of this work is to examine these quantities employing cutoff regularization schemes.

Another complication arises from the closeness of the spin-3/2 decuplet resonances which are separated only by $231 \mathrm{MeV}$ in average from the octet baryons which is considerably smaller than the kaon and the $\eta$ mass. These resonances are, therefore, expected to play an important part at low energies. It has been suggested [4] to include the decuplet explicitely.

The present work is organized as follows. In the next section we apply two different cutoff schemes to regularize the Feynman diagrams without decuplet contributions. Besides the dipole cutoff already used in [2, 3] we consider a slightly modified dipole cutoff which is identical to the first one for vanishing off-shell momenta of the baryons. The following section deals with the inclusion of the decuplet fields. The results for both cases are presented in Sec. 4. In Sec. 5 we conclude with a short summary. The decuplet contributions to the scalar form factors and the octet baryon masses are relegated to the Appendices.

\section{$2 \sigma$-terms in a cutoff scheme}

In this section, we will work with the heavy baryon Lagrangian for the Goldstone bosons and the octet baryons which can be decomposed into a lowest order and a next-to-leading order part in the derivative expansion

$$
\mathcal{L}_{\phi B}=\mathcal{L}_{\phi B}^{(1)}+\mathcal{L}_{\phi B}^{(2)}
$$

where the superscript denotes the chiral order. In the heavy baryon formulation the baryons are described by a four-velocity $v_{\mu}$ and relativistic corrections appear as $1 / \stackrel{\circ}{M}$ corrections where $\stackrel{\circ}{M}$ is the average octet baryon mass in the chiral limit. A consistent chiral counting scheme emerges, i.e. a one-to-one correspondence between the Goldstone boson loops and the expansion in small 
momenta and quark masses. The lowest order Lagrangian $\mathcal{L}_{\phi B}^{(1)}$ includes the two axial-vector couplings $D$ and $F$

$$
\mathcal{L}_{\phi B}^{(1)}=i \operatorname{tr}(\bar{B}[v \cdot D, B])+D \operatorname{tr}\left(\bar{B} S_{\mu}\left\{u^{\mu}, B\right\}\right)+F \operatorname{tr}\left(\bar{B} S_{\mu}\left[u^{\mu}, B\right]\right)
$$

where $2 S_{\mu}=i \gamma_{5} \sigma_{\mu \nu} v^{\nu}$ denotes the Pauli-Lubanski spin vector. The pseudoscalar Goldstone fields $(\phi=\pi, K, \eta)$ are collected in the $3 \times 3$ unimodular, unitary matrix $U(x)$,

$$
U(\phi)=u^{2}(\phi)=\exp \{2 i \phi / \stackrel{\circ}{F}\}
$$

with $\stackrel{\circ}{F}$ being the pseudoscalar decay constant (in the chiral limit), and

$$
\phi=\frac{1}{\sqrt{2}}\left(\begin{array}{ccc}
\frac{1}{\sqrt{2}} \pi^{0}+\frac{1}{\sqrt{6}} \eta & \pi^{+} & K^{+} \\
\pi^{-} & -\frac{1}{\sqrt{2}} \pi^{0}+\frac{1}{\sqrt{6}} \eta & K^{0} \\
K^{-} & \bar{K}^{0} & -\frac{2}{\sqrt{6}} \eta
\end{array}\right) .
$$

Under $\mathrm{SU}(3)_{L} \times \mathrm{SU}(3)_{R}, U(x)$ transforms as $U \rightarrow U^{\prime}=L U R^{\dagger}$, with $L, R \in \mathrm{SU}(3)_{L, R}$. One forms an object of axial-vector type with one derivative

$$
u_{\mu}=i u^{\dagger} \nabla_{\mu} U u^{\dagger}
$$

with $\nabla_{\mu}$ being the covariant derivative of $U$. The matrix $B$ denotes the baryon octet,

$$
B=\left(\begin{array}{ccc}
\frac{1}{\sqrt{2}} \Sigma^{0}+\frac{1}{\sqrt{6}} \Lambda & \Sigma^{+} & p \\
\Sigma^{-} & -\frac{1}{\sqrt{2}} \Sigma^{0}+\frac{1}{\sqrt{6}} \Lambda & n \\
\Xi^{-} & \Xi^{0} & -\frac{2}{\sqrt{6}} \Lambda
\end{array}\right) .
$$

The matrices $u_{\mu}$ and $B$ transform under $S U(3)_{L} \times S U(3)_{R}$ as any matter field, e.g.,

$$
B \rightarrow B^{\prime}=K B K^{\dagger}
$$

with $K(U, L, R)$ the compensator field representing an element of the conserved subgroup $\mathrm{SU}(3)_{V}$. We will use $D=3 / 4$ and $F=1 / 4$ which leads to $g_{A}=D+F=1.25$. At next-to-leading order explicit chiral symmetry breaking terms appear

$$
\mathcal{L}_{\phi B}^{(2)}=b_{0} \operatorname{tr}(\bar{B} B) \operatorname{tr}\left(\chi_{+}\right)+b_{D} \operatorname{tr}\left(\bar{B}\left\{\chi_{+}, B\right\}\right)+b_{F} \operatorname{tr}\left(\bar{B}\left[\chi_{+}, B\right]\right)
$$

with $\chi_{+}=2 B_{0}\left(u^{\dagger} \mathcal{M} u^{\dagger}+u \mathcal{M} u\right)$ and $\mathcal{M}=\operatorname{diag}\left(m_{u}, m_{d}, m_{s}\right)$ the quark mass matrix. We prefer to work in the isospin limit $m_{u}=m_{d}=\hat{m}$. To this order three coupling constant, so called low-energy constants (LECs), appear. Together with the average octet baryon mass in the chiral limit $\stackrel{\circ}{M}$ we end up with four unknown parameters. These have to be fixed from phenomenology. Here we will use the four different masses for the octet baryons in the isospin limit. Since both $\stackrel{\circ}{M}$ and $b_{0}$ shift the baryon mass spectrum by a constant the pion nucleon sigma-term at zero momentum transfer $\sigma_{\pi N}(0)$ ( or one of the kaon nucleon sigma-terms ) has also to be taken into account in order to fix all parameters. 
One defines the scalar form factors or $\sigma$-terms which measure the strength of $m_{q} \bar{q} q$ in the proton by

$$
\begin{aligned}
\sigma_{\pi N}(t) & \left.=\hat{m}<p^{\prime}|\bar{u} u+\bar{d} d| p\right\rangle, \\
\sigma_{K N}^{(1)}(t) & =\frac{1}{2}\left(\hat{m}+m_{s}\right)<p^{\prime}|\bar{u} u+\bar{s} s| p>, \\
\sigma_{K N}^{(2)}(t) & =\frac{1}{2}\left(\hat{m}+m_{s}\right)<p^{\prime}|-\bar{u} u+2 \bar{d} d+\bar{s} s| p>,
\end{aligned}
$$

with $\mid p>$ a proton state with four-momentum $p, t=\left(p^{\prime}-p\right)^{2}$ the invariant momentum transfer squared. It is most convenient to work in the Breit-frame in which $v \cdot p^{\prime}=v \cdot p$. There are two types of contributions for the $\sigma$-terms. To lowest order, there are the tree level contributions of chiral order two from the counterterms $b_{0}, b_{D}, b_{F}$ of the Lagrangian $\mathcal{L}_{\phi B}^{(2)}$. The contributions from the Goldstone boson loops appear at next-to-leading order and can be evaluated by using dimensional regularization [5]. A typical integral in this analysis has for the case of zero momentum transfer and $d$ dimensions the form

$$
\int \frac{d^{d} l}{(2 \pi)^{d}} \frac{i^{3}(S \cdot l)^{2}}{\left[l^{2}-m_{\phi}^{2}+i \epsilon\right]^{2}[v \cdot l+i \epsilon]}=\frac{3}{64 \pi} m_{\phi}
$$

where $m_{\phi}$ is the meson mass. The result is non-analytic in the quark masses since $m_{\phi} \propto m_{q}^{1 / 2}$. The integral grows linearly with increasing meson mass. We expect the long distance portion of the integral to be larger for small meson masses since for small momenta the meson propagator can be approximated by $1 / m_{\phi}^{2}$. This indicates that in the dimensionally regularized integral there are significant contributions from short distance physics which cannot be described appropriately by chiral symmetry. Therefore, one has to employ other regularization schemes that emphasize long distance effects of the integrals and reduce short distance contributions. In [3] it was shown that a simple dipole regulator fulfills these requirements.

For the evaluation of the Goldstone boson loops we will employ the regulators

$$
R_{1}=\left(\frac{\Lambda^{2}}{\Lambda^{2}-l^{2}}\right)^{2} \quad, \quad R_{2}=\left(\frac{\Lambda^{2}}{\Lambda^{2}-l^{2}}\right)\left(\frac{\Lambda^{2}}{\Lambda^{2}-(l+q)^{2}}\right)
$$

where $l$ is the loop momentum and $q$ the small off-shell momentum of the external baryons in the heavy mass formalism. Both cases are identical for vanishing off-shell momentum $q$. The reason for considering the regulator $R_{2}$ in addition to $R_{1}$ will become clear when we introduce decuplet fields. It turns out that including decuplet fields leads to divergent integrals in the case of the regulator $R_{1}$, whereas $R_{2}$ avoids this difficulty. Also, we are able to compare the results for the $\sigma$-terms in both regularization schemes and examine the dependence on employing different regulators. Inserting these regulators into the integral in Eq. (9) leads to

$$
I_{\Lambda}=\int \frac{d^{d} l}{(2 \pi)^{d}} \frac{i^{3}(S \cdot l)^{2}}{\left[l^{2}-m_{\phi}^{2}+i \epsilon\right]^{2}[v \cdot l+i \epsilon]}\left(\frac{\Lambda^{2}}{\Lambda^{2}-l^{2}}\right)^{2}=-\frac{1}{64 \pi} \frac{\Lambda^{4}}{\left(\Lambda+m_{\phi}\right)^{3}}
$$

The introduction of the additional scale $\Lambda$ spoilt the one-to-one correspondence between the meson loops and the expansion in the quark masses and the integral depends strongly on the value of the cutoff $\Lambda$. However, this does not mean that to the order we are working the resulting 
physics will depend on $\Lambda$, since one is able to absorb the effects of $\Lambda$ into a renormalization of the LECs. To this end, one expands the result in Eq. (11) in terms of the meson mass $m_{\phi}$

$$
I_{\Lambda} \stackrel{m_{\phi}<<\Lambda}{\longrightarrow}-\frac{1}{64 \pi} \Lambda+\frac{3}{64 \pi} m_{\phi}+\ldots
$$

where the ellipsis stands for higher orders in $m_{\phi}$. The second term in the expansion agrees with the result from the dimensionally regularized version. The first term is a constant and can be absorbed into renormalizations of the coefficients $b_{0}, b_{D}, b_{F}$ of the Lagrangian $\mathcal{L}_{\phi B}^{(2)}$, and indeed this is found to be the case - one verifies that

$$
\begin{aligned}
& b_{D}^{r}=b_{D}-\frac{3 F^{2}-D^{2}}{128 \pi \stackrel{\circ}{F}^{2}} \Lambda \\
& b_{F}^{r}=b_{F}-\frac{5 D F}{192 \pi \stackrel{\circ}{F}^{2}} \Lambda \\
& b_{0}^{r}=b_{0}-\frac{13 D^{2}+9 F^{2}}{576 \pi \stackrel{\circ}{F}^{2}} \Lambda
\end{aligned}
$$

which agrees with the result already obtained in the analysis for the baryon masses [3]. That this renormalization can occur involves a highly constrained set of conditions and the fact that they are satisfied is a significant verification of the chiral invariance of the cutoff procedure.

The chiral expansions at next-to-leading order for the $\sigma$-terms read for zero momentum transfer

$$
\begin{aligned}
\sigma_{\pi N}(0)= & \frac{m_{\pi}^{2}}{64 \pi F_{\pi}^{2}} \Lambda^{4}\left(3[D+F]^{2} \frac{1}{\left(\Lambda+m_{\pi}\right)^{3}}+\frac{1}{3}\left[5 D^{2}-6 D F+9 F^{2}\right] \frac{1}{\left(\Lambda+m_{K}\right)^{3}}\right. \\
& \left.+\frac{1}{9}[D-3 F]^{2} \frac{1}{\left(\Lambda+m_{\eta}\right)^{3}}\right)-2 m_{\pi}^{2}\left(b_{D}+b_{F}+2 b_{0}\right) \\
\sigma_{K N}^{(1)}(0)= & \frac{m_{K}^{2}}{64 \pi F_{\pi}^{2}} \Lambda^{4}\left(\frac{3}{2}[D+F]^{2} \frac{1}{\left(\Lambda+m_{\pi}\right)^{3}}+\left[\frac{7}{3} D^{2}-2 D F+5 F^{2}\right] \frac{1}{\left(\Lambda+m_{K}\right)^{3}}\right. \\
& \left.+\frac{5}{18}[D-3 F]^{2} \frac{1}{\left(\Lambda+m_{\eta}\right)^{3}}-\frac{1}{3}[D-3 F][D+F] \frac{\left(m_{\pi}+m_{\eta}\right) \Lambda+2 m_{\pi} m_{\eta}}{\left[\Lambda+m_{\pi}\right]^{2}\left[\Lambda+m_{\eta}\right]^{2}\left[m_{\pi}+m_{\eta}\right]}\right) \\
& -4 m_{K}^{2}\left(b_{D}+b_{0}\right) \\
\sigma_{K N}^{(2)}(0)= & \frac{m_{K}^{2}}{64 \pi F_{\pi}^{2}} \Lambda^{4}\left(\frac{3}{2}[D+F]^{2} \frac{1}{\left(\Lambda+m_{\pi}\right)^{3}}+3[D-F]^{2} \frac{1}{\left(\Lambda+m_{K}\right)^{3}}\right. \\
& \left.+\frac{5}{18}[D-3 F]^{2} \frac{1}{\left(\Lambda+m_{\eta}\right)^{3}}+[D-3 F][D+F] \frac{\left(m_{\pi}+m_{\eta}\right) \Lambda+2 m_{\pi} m_{\eta}}{\left[\Lambda+m_{\pi}\right]^{2}\left[\Lambda+m_{\eta}\right]^{2}\left[m_{\pi}+m_{\eta}\right]}\right) \\
& -4 m_{K}^{2}\left(b_{0}-b_{F}\right)
\end{aligned}
$$

where we have replaced $\stackrel{\circ}{F}$ by the pion decay constant $F_{\pi}=93 \mathrm{MeV}$, which is legitimate to the order we are working. Note that an additional contribution arises for the two kaon nucleon $\sigma$-terms from the $\pi^{0} \eta$ loop. Furthermore, the $\pi N \sigma$-term is related to the nucleon mass by the Feynman-Hellman theorem $\sigma_{\pi N}(0)=\hat{m}\left(\partial m_{N} / \partial \hat{m}\right)$. 
The strange contribution to the nucleon mass is given by the $\sigma$-terms at zero momentum transfer

$$
m_{s}<p|\bar{s} s| p>=\left(\frac{1}{2}-\frac{m_{\pi}^{2}}{4 m_{K}^{2}}\right)\left(3 \sigma_{K N}^{(1)}(0)+\sigma_{K N}^{(2)}(0)\right)+\left(\frac{1}{2}-\frac{m_{K}^{2}}{m_{\pi}^{2}}\right) \sigma_{\pi N}(0) .
$$

This matrix element can be deduced by means of the Feynman-Hellman theorem $<p|\bar{s} s| p>=$ $\partial m_{N} / \partial m_{s}$. The strangeness fraction $y$ of the nucleon is given by

$$
y=\frac{2<p|\bar{s} s| p>}{<p|\bar{u} u+\bar{d} d| p>}=\frac{m_{\pi}^{2}}{\sigma_{\pi N}(0)}\left(m_{K}^{2}-\frac{1}{2} m_{\pi}^{2}\right)^{-1} m_{s}<p|\bar{s} s| p>
$$

and one defines the quantity $\hat{\sigma}$ via

$$
\sigma_{\pi N}(0)=\frac{\hat{\sigma}}{1-y}
$$

For non-vanishing $t$ we have to distinguish between both regularization schemes $R_{1}$ and $R_{2}$. The general formulae for the scalar form factors read

$$
\begin{aligned}
\sigma_{\pi N}(t)= & \frac{m_{\pi}^{2}}{64 \pi F_{\pi}^{2}} \Lambda^{4}\left(3[D+F]^{2} J^{i}\left(m_{\pi}\right)+\frac{1}{3}\left[5 D^{2}-6 D F+9 F^{2}\right] J^{i}\left(m_{K}\right)\right. \\
& \left.+\frac{1}{9}[D-3 F]^{2} J^{i}\left(m_{\eta}\right)\right)-2 m_{\pi}^{2}\left(b_{D}+b_{F}+2 b_{0}\right) \\
\sigma_{K N}^{(1)}(t)= & \frac{m_{K}^{2}}{64 \pi F_{\pi}^{2}} \Lambda^{4}\left(\frac{3}{2}[D+F]^{2} J^{i}\left(m_{\pi}\right)+\left[\frac{7}{3} D^{2}-2 D F+5 F^{2}\right] J^{i}\left(m_{K}\right)\right. \\
& \left.+\frac{5}{18}[D-3 F]^{2} J^{i}\left(m_{\eta}\right)-\frac{1}{6}[D-3 F][D+F] K^{i}\right)-4 m_{K}^{2}\left(b_{D}+b_{0}\right) \\
\sigma_{K N}^{(2)}(t)= & \frac{m_{K}^{2}}{64 \pi F_{\pi}^{2}} \Lambda^{4}\left(\frac{3}{2}[D+F]^{2} J^{i}\left(m_{\pi}\right)+3[D-F]^{2} J^{i}\left(m_{K}\right)+\frac{5}{18}[D-3 F]^{2} J^{i}\left(m_{\eta}\right)\right. \\
& \left.+\frac{1}{2}[D-3 F][D+F] K^{i}\right)-4 m_{K}^{2}\left(b_{0}-b_{F}\right)
\end{aligned}
$$

with $i=1,2$. Employing the regulator $R_{1}$ for the evaluation of the Goldstone boson loops leads to

$$
\begin{aligned}
J^{1}\left(m_{\phi}\right)= & -\frac{1}{\left(m_{\phi}^{2}-\Lambda^{2}\right)^{2}}\left(m_{\phi}-\Lambda-\frac{m_{\phi}\left(m_{\phi}^{2}-\Lambda^{2}\right)}{\left(m_{\phi}+\Lambda\right)^{2}-t}\right. \\
& \left.+\frac{m_{\phi}^{2}-\frac{1}{2} t}{\sqrt{t}}\left[\ln \frac{2 m_{\phi}+\sqrt{t}}{2 m_{\phi}-\sqrt{t}}-\ln \frac{m_{\phi}+\Lambda+\sqrt{t}}{m_{\phi}+\Lambda-\sqrt{t}}\right]\right) \\
K^{1}= & -\frac{1}{\left(m_{\eta}^{2}-\Lambda^{2}\right)^{2}}\left(m_{\eta}-\Lambda-\frac{m_{\pi}\left(m_{\eta}^{2}-\Lambda^{2}\right)}{\left(m_{\pi}+\Lambda\right)^{2}-t}\right. \\
& \left.\quad+\frac{m_{\pi}^{2}+m_{\eta}^{2}-t}{2 \sqrt{t}}\left[\ln \frac{m_{\pi}+m_{\eta}+\sqrt{t}}{m_{\pi}+m_{\eta}-\sqrt{t}}-\ln \frac{m_{\pi}+\Lambda+\sqrt{t}}{m_{\pi}+\Lambda-\sqrt{t}}\right]\right) \\
& -\frac{1}{\left(m_{\pi}^{2}-\Lambda^{2}\right)^{2}}\left(m_{\pi}-\Lambda-\frac{m_{\eta}\left(m_{\pi}^{2}-\Lambda^{2}\right)}{\left(m_{\eta}+\Lambda\right)^{2}-t}\right. \\
& \left.\quad+\frac{m_{\eta}^{2}+m_{\pi}^{2}-t}{2 \sqrt{t}}\left[\ln \frac{m_{\pi}+m_{\eta}+\sqrt{t}}{m_{\pi}+m_{\eta}-\sqrt{t}}-\ln \frac{m_{\eta}+\Lambda+\sqrt{t}}{m_{\eta}+\Lambda-\sqrt{t}}\right]\right),
\end{aligned}
$$


whereas for the modified regulator $R_{2}$ one obtains

$$
\begin{aligned}
J^{2}\left(m_{\phi}\right)= & -\frac{1}{\left(m_{\phi}^{2}-\Lambda^{2}\right)^{2}} \frac{1}{\sqrt{t}}\left(\left[m_{\phi}^{2}-\frac{1}{2} t\right] \ln \frac{2 m_{\phi}+\sqrt{t}}{2 m_{\phi}-\sqrt{t}}\right. \\
& \left.+\left[\Lambda^{2}-\frac{1}{2} t\right] \ln \frac{2 \Lambda+\sqrt{t}}{2 \Lambda-\sqrt{t}}-\left[m_{\phi}^{2}+\Lambda^{2}-t\right] \ln \frac{m_{\phi}+\Lambda+\sqrt{t}}{m_{\phi}+\Lambda-\sqrt{t}}\right) \\
K^{2}= & -\frac{1}{\left(m_{\eta}^{2}-\Lambda^{2}\right)\left(m_{\pi}^{2}-\Lambda^{2}\right)} \frac{1}{\sqrt{t}}\left(\left[m_{\pi}^{2}+m_{\eta}^{2}-t\right] \ln \frac{m_{\pi}+m_{\eta}+\sqrt{t}}{m_{\pi}+m_{\eta}-\sqrt{t}}\right. \\
& -\left[m_{\pi}^{2}+\Lambda^{2}-t\right] \ln \frac{m_{\pi}+\Lambda+\sqrt{t}}{m_{\pi}+\Lambda-\sqrt{t}}-\left[m_{\eta}^{2}+\Lambda^{2}-t\right] \ln \frac{m_{\eta}+\Lambda+\sqrt{t}}{m_{\eta}+\Lambda-\sqrt{t}} \\
& \left.+\left[2 \Lambda^{2}-t\right] \ln \frac{2 \Lambda+\sqrt{t}}{2 \Lambda-\sqrt{t}}\right) .
\end{aligned}
$$

One is in particular interested in the shifts of the $\sigma$-terms to the Cheng-Dashen points. These are $t=2 m_{\pi}^{2}$ and $t=2 m_{K}^{2}$ for the $\pi N$ and $K N \sigma$-terms, respectively. The $\sigma$-terms can acquire imaginary parts depending on the values for $t$ and $\Lambda$. Since we will consider $\Lambda$ only in the range from $300 \mathrm{MeV}$ to $600 \mathrm{Mev}$ the shift of the $\pi N \sigma$-term is real. On the other hand, the shifts of the two $K N \sigma$-terms acquire an imaginary part at $t=4 m_{\pi}^{2}$ and also at $t=\left(m_{\pi}+\Lambda\right)^{2}$ for sufficiently small values of $\Lambda$ in both regularization schemes. In the regularization scheme $R_{2}$ there is an additional branch cut starting at $t=4 \Lambda^{2}$. For increasing $\Lambda$ only the first branch cut $t=4 m_{\pi}^{2}$ from the pion loop remains. This agrees with the dimensional regularization scheme which is recovered for $\Lambda \rightarrow \infty$. Since we choose the Gell-Mann-Okubo value for the $\eta$ mass, the $\pi \eta$ loop does not acquire an imaginary part below $t=2 m_{K}^{2}<\left(m_{\pi}+m_{\eta}\right)^{2}$. For the physical mass of the $\eta$ this contribution is tiny compared to the other parts. Before presenting the numerical results we will include the decuplet in the next section.

\section{Inclusion of the decuplet}

In general it is assumed that baryon resonance states are much heavier compared to the lowestlying baryon octet. In this case they can be integrated out and replaced by counterterms that do not include these resonance states explicitely. However, while this might be a reasonable procedure for heavier resonances like the Roper-octet, it is a questionable assumption for the decuplet. The low-lying decuplet is separated from the octet by only $\Delta=231 \mathrm{MeV}$ in average which is much smaller than the $K$ or the $\eta$ mass. Furthermore, the $\Delta(1232)$ couples strongly to the $\pi N$ sector and its contribution plays an important role in the channels wherein this effect is possible. In the meson sector, the first resonance is the vector meson $\rho$ with a mass of 770 $\mathrm{MeV}$ which is considerably heavier than the Goldstone bosons. It was therefore argued in [4] to include the spin-3/2 decuplet as explicit degrees of freedom. In the framework of conventional heavy baryon CHPT it was found that intermediate $\Delta(1232)$ states give a contribution of 7.5 $\mathrm{MeV}$ to the $\pi N \sigma$-term shift which is as large as the contribution from the the octet alone [5].

In this section we will include the decuplet fields and the resulting loop integrals are evaluated by using the regulator $R_{2}$. A similar analysis with the regulator $R_{1}$ instead is not possible

since then divergent integrals arise for non-vanishing momentum $t$. The pertinent interaction 
Lagrangian between the spin-3/2 fields - denoted by the Rarita-Schwinger fields $T_{\mu}-$, the baryon octet and the Goldstone bosons reads

$$
\mathcal{L}_{\phi B T}=-i \bar{T}^{\mu} v \cdot D T_{\mu}+\Delta \bar{T}^{\mu} T_{\mu}+\frac{C}{2}\left(\bar{T}^{\mu} u_{\mu} B+\bar{B} u_{\mu} T^{\mu}\right)
$$

where we have suppressed the flavor $S U(3)$ indices. In the heavy mass formulation the fields $T_{\mu}$ satisfy the condition $v \cdot T=0$. The coupling constant $C=1.2 \ldots 1.8$ can be determined from the strong decays $T \rightarrow B \pi$. After integrating out the heavy degrees of freedom from the relativistic Lagrangian there is still a remaining mass dependence which is proportional to the average octet-decuplet splitting $\Delta$ and does not vanish in the chiral limit. In the Feynman rules the mass splitting $\Delta$ is contained in the decuplet propagator

$$
\frac{i}{v \cdot l-\Delta+i \epsilon}\left(v_{\mu} v_{\nu}-g_{\mu \nu}-\frac{4}{3} S_{\mu} S_{\nu}\right)
$$

The appearance of the mass scale $\Delta$ destroys in the case of dimensional regularization the oneto-one correspondence between meson loops and the expansion in small momenta and quark masses. No further complications arise in our case since the strict chiral counting scheme has already been spoilt by introducing the scale $\Lambda$.

For zero momentum transfer $t=0$ the decuplet contributions to the $\sigma$-terms read

$$
\begin{aligned}
\delta \sigma_{\pi N}(0) & =-\frac{m_{\pi}^{2} C^{2}}{96 \pi F_{\pi}^{2}} \Lambda^{4}\left(8 H\left(m_{\pi}\right)+H\left(m_{K}\right)\right) \\
\delta \sigma_{K N}^{(1)}(0) & =-\frac{m_{K}^{2} C^{2}}{96 \pi F_{\pi}^{2}} \Lambda^{4}\left(4 H\left(m_{\pi}\right)+\frac{4}{3} H\left(m_{K}\right)\right) \\
\delta \sigma_{K N}^{(2)}(0) & =-\frac{m_{K}^{2} C^{2}}{96 \pi F_{\pi}^{2}} \Lambda^{4}\left(4 H\left(m_{\pi}\right)+2 H\left(m_{K}\right)\right)
\end{aligned}
$$

with

$$
\begin{array}{r}
H\left(m_{\phi}\right)=\frac{1}{\left(\Lambda^{2}-m_{\phi}^{2}\right)^{3}}\left(-\Delta\left[\Lambda^{2}-m_{\phi}^{2}\right]+\frac{1}{2} \Delta\left[4 \Delta^{2}-3 \Lambda^{2}-3 m_{\phi}^{2}\right] \ln \frac{m_{\phi}^{2}}{\Lambda^{2}}\right. \\
+\left[4 \Delta^{2}-3 \Lambda^{2}-m_{\phi}^{2}\right] \sqrt{\Delta^{2}-m_{\phi}^{2}} \ln \left[\frac{\Delta}{m_{\phi}}+\sqrt{\left.\frac{\Delta^{2}}{m_{\phi}^{2}}-1\right]}\right. \\
\left.+\left[4 \Delta^{2}-\Lambda^{2}-3 m_{\phi}^{2}\right] \sqrt{\Lambda^{2}-\Delta^{2}} \arccos \frac{\Delta}{\Lambda}\right) ; \text { for } m_{\phi}<\Delta \\
H\left(m_{\phi}\right)=\frac{1}{\left(\Lambda^{2}-m_{\phi}^{2}\right)^{3}}\left(-\Delta\left[\Lambda^{2}-m_{\phi}^{2}\right]+\frac{1}{2} \Delta\left[4 \Delta^{2}-3 \Lambda^{2}-3 m_{\phi}^{2}\right] \ln \frac{m_{\phi}^{2}}{\Lambda^{2}}\right. \\
-\left[4 \Delta^{2}-3 \Lambda^{2}-m_{\phi}^{2}\right] \sqrt{m_{\phi}^{2}-\Delta^{2}} \arccos \frac{\Delta}{m_{\phi}}
\end{array}
$$

where we required $\Lambda>\Delta$. For the limit $\Lambda>m_{\phi}$ we recover up to some constant terms the result from dimensional regularization [5]

$$
H\left(m_{\phi}\right) \stackrel{m_{\phi}<<\Lambda}{\longrightarrow}-\frac{3}{2}\left(\Delta\left[\ln \frac{m_{\phi}^{2}}{\Lambda^{2}}+\frac{2}{3}\right]-2 \sqrt{m_{\phi}^{2}-\Delta^{2}} \arccos \frac{\Delta}{m_{\phi}}+\frac{\pi}{3} \Lambda+\ldots\right)
$$


for the case $m_{\phi}>\Delta$ and an analogous result for $m_{\phi}<\Delta$. The constant terms can again be absorbed into a renormalization of the parameters $b_{0, D, F}$. The results for the scalar form factors for general $t$ can be found in App. A.

\section{Results and discussion}

In this section we present the numerical results for the calculation of the $\sigma$-terms. We consider first the case with no resonances. The values for our parameters are $D=0.75, F=0.5, F_{\pi}=93$ $\mathrm{MeV}, m_{\pi}=138 \mathrm{MeV}, m_{K}=495 \mathrm{MeV}$, and for the mass of the $\eta$ we use the GMO value for the pseudoscalar mesons $m_{\eta}=566 \mathrm{MeV}$. The differences for $F_{\pi}$ and $m_{\eta}$ to $\stackrel{\circ}{F}$-the pseudoscalar decay constant in the chiral limit- and to the physical mass of $\eta$, respectively, appear only at higher orders. We will restrict ourselves to these central values of the parameters since a small variation in these parameters does only lead to some minor changes in the results.

In baryon chiral perturbation theory, the transition between short and long distance occurs around a distance scale of $\sim 1$ fermi, or a momentum scale of $\sim 200 \mathrm{MeV}$. This corresponds to the measured size of a baryon. The effective field theory treats the baryons and pions as point particles. This is appropriate for the very long distance physics. However, for propagation at distances less then the separation scale, the point particle theory is not an accurate representation of the physics. The composite substructure becomes manifest below this point.

Of course, the cutoff $\Lambda$ should not be taken so low in energy that it removes any truly long distance physics. Also, while it can in principle be taken much larger than the separation scale, this will lead to the inclusion of spurious short distance physics which can upset the convergence of the expansion. It is ideal to take the cutoff slightly above the separation scale so that all of the long distance physics, but little of the short distance physics, is included. Therefore, we will vary the cutoff in the range $\Lambda \geq 1 /<r_{B}>\sim 300-600 \mathrm{MeV}$.

The four unknown parameters $b_{0, D, F}$ and $\stackrel{\circ}{M}$ have to be fixed from phenomenology. We will choose the four different baryon masses in the isospin limit and the value of $\sigma_{\pi N} \simeq 45 \mathrm{MeV}$ to perform a least-squares fit for these parameters. The explicit calculation of the masses in the cutoff regularization scheme to the order we are working can be found in $[3 \|$. As outlined in this work the asymptotic mass-independent component of the mass integral which is proportional to $\Lambda^{3}$ is removed by redefining $\stackrel{\circ}{M}$

$$
\stackrel{\circ}{M}_{r}=\stackrel{\circ}{M}-\left(5 D^{2}+9 F^{2}\right) \frac{\Lambda^{3}}{48 \pi F_{\pi}^{2}} .
$$

In Tables 1 and 3 we present the renormalized values $\stackrel{\circ}{M}_{r}$ after absorbing the consatnt pieces from the mass integral. We are then able to make predictions for the $K N \sigma$-terms and for the shifts to the Cheng-Dashen points. The results of this calculation are shown in Tab. 1 and Tab. 2. In the first Table, we present besides the values for $b_{0, D, F}$ and $\stackrel{\circ}{M}$ the two $K N \sigma$-terms at zero momentum transfer, the strange quark matrix element from Eq. (17), the strange quark fraction $y, \hat{\sigma}$ and the deviation from the Gell-Mann-Okubo relation $\left[3 M_{\Lambda}+M_{\Sigma}-2 M_{N}-2 M_{\Xi}\right] / 4$ between the baryon masses which is experimentally about $6.5 \mathrm{MeV}$. Within the accuracy of the calculation, the $K N \sigma$-terms turn out to be

$$
\sigma_{K N}^{(1)}(0)=400 \pm 30 \mathrm{MeV}
$$




$$
\sigma_{K N}^{(2)}(0)=270 \pm 20 \mathrm{MeV}
$$

where the uncertainty stems from the variation in the cutoff $\Lambda$. For the other quantities we have

$$
m_{s}<p|\bar{s} s| p>=150 \pm 50 \mathrm{MeV}, \quad y=0.25 \pm 0.05, \quad \hat{\sigma}=33 \pm 3 \mathrm{MeV}
$$

The value for $y$ is within the band deduced in [6], $y=0.15 \pm 0.10$, and the value for $\hat{\sigma}$ agrees nicely with Gasser's estimate $\hat{\sigma}=33 \pm 5 \mathrm{MeV}$, given in [7]. Therein, the author comes to the conclusion that the lowest non-analytic corrections to the baryon masses and the $\pi N \sigma$-term are so large, that chiral perturbation theory is meaningless in that case. He proposes a meson-cloud model by introducing a cutoff which regularizes the divergent integrals. The cutoff is provided by the square of the axial vector form factor which enters the expression of the propagator and is similar to $R_{1}^{2}$ in our notation. The deviation from the Gell-Mann-Okubo relation due to loops is found to be quite small in dimensional regularization, primarily due to the (accidental) feature that it is proportional to $D^{2}-3 F^{2}<<1$. We, therefore, expect this deviation to be even smaller in the cutoff scheme. The chiral expansions of the $\sigma$-terms in dimensional regularization read

$$
\begin{aligned}
\sigma_{\pi N}(0) & =82.7-37.7 \mathrm{MeV}=45.0 \mathrm{MeV} \\
\sigma_{K N}^{(1)}(0) & =763.0-605.6 \mathrm{MeV}=157.4 \mathrm{MeV} \\
\sigma_{K N}^{(2)}(0) & =177.1-56.6 \mathrm{MeV}=120.5 \mathrm{MeV} .
\end{aligned}
$$

The chiral expansions of the $\sigma$-terms in the cutoff scheme for $\Lambda=400 \mathrm{MeV}$ are

$$
\begin{aligned}
& \sigma_{\pi N}(0)=36.2+8.8 \mathrm{MeV}=\quad 45.0 \mathrm{MeV} \\
& \sigma_{K N}^{(1)}(0)=340.5+66.6 \mathrm{MeV}=407.1 \mathrm{MeV} \\
& \sigma_{K N}^{(2)}(0)=227.0+48.1 \mathrm{MeV}=275.1 \mathrm{MeV}
\end{aligned}
$$

where the first number denotes the lowest order contribution from the tree level result and the second number the Goldstone boson loop contribution. The chiral expansions in the cutoff scheme are much improved with respect to the case of dimensional regularization, especially for $\sigma_{\pi N}$ and $\sigma_{K N}^{(1)}$. While in dimensional regularization the contributions of two successive chiral orders are of opposite sign and tend to cancel each other - a common feature in this regularization scheme -, there is a clear convergence in the cutoff scheme. We also examined our results by varying the value of the $\pi N \sigma$-term by $\pm 10 \mathrm{MeV}$. This change alters the value of $b_{0}$ from -0.29 to -0.55 $\mathrm{GeV}^{-1}$ which has a quite dramatic impact on the $K N \sigma$-terms and the value of $m_{s}<p|\bar{s} s| p>$. This is in agreement with the calculation in dimensional regularization [5]. We will restrict ourselves to the central value of $\sigma_{\pi N}=45 \mathrm{MeV}$ [6] in our analysis. The changes in a calculation with the physical mass of the $\eta, m_{\eta}=549 \mathrm{MeV}$, are negligible.

In Table 2 we list the shifts of the scalar form factors to the Cheng-Dashen points. We are able to compare both regularization schemes $R_{1}$ and $R_{2}$ since these differ only for non-vanishing offshell momenta of the baryons. While there is agreement for the $\pi N \sigma$-term, both regularization schemes differ considerably in the $K N \sigma$-terms which depend strongly on the cutoff $\Lambda$. This might indicate that for these quantities higher orders play an essential role. Clearly a definite statement about the shifts to the Cheng-Dashen points for the $K N \sigma$-terms cannot be made. The $\pi N \sigma$-term shift agrees in both regularization schemes and we find

$$
\sigma_{\pi N}\left(2 m_{\pi}^{2}\right)-\sigma_{\pi N}(0)=4 \pm 1 \mathrm{MeV}
$$


This value is in agreement with the result $\Delta \sigma_{\pi N}=5 \pm 1 \mathrm{MeV}$ of the complete fourth order calculation in conventional heavy baryon chiral perturbation theory [8]. On the other hand, it is smaller than the empirical value found in [6]. The main contribution to the $\pi N \sigma$-term shift in the dispersive calculation in that paper comes from an energy region in which the one-loop approximation is off by a factor of two. Therefore, we expect contributions from higher chiral orders to be significant. It remains to be seen how higher order corrections not yet accounted for will modify Eq. (37).

Adding the decuplet, we set $\Delta=231 \mathrm{MeV}$, which is the average octet-decuplet mass splitting, and the value of the coupling constant $C$ is given by $C=1.5$ from an overall fit to the decuplet decays 99. To perform a least-squares fit for the parameters $b_{0, D, F}$ and $\stackrel{\circ}{M}$ we have to include the decuplet contributions to the octet baryon masses, see App. B. The results of the fit can be found in Tab. 3 and Tab. 4. We absorbed again the asymptotic mass-independent component of the mass integral by redefining $\stackrel{\circ}{M}$

$$
\stackrel{\circ}{M_{r}} \rightarrow \stackrel{\circ}{M}_{r}-\frac{5 C^{2} \Lambda^{2}}{24 \pi^{2} F_{\pi}^{2}}\left(-\frac{\pi}{4} \Lambda+\frac{1}{2} \Delta\right)
$$

It turns out that there are no significant changes in the results as in the case of dimensional regularization. We obtain for the $K N \sigma$-terms

$$
\begin{aligned}
& \sigma_{K N}^{(1)}(0)=380 \pm 40 \mathrm{MeV} \\
& \sigma_{K N}^{(2)}(0)=250 \pm 30 \mathrm{MeV} .
\end{aligned}
$$

For the strange quark contribution to the nucleon we have

$$
m_{s}<p|\bar{s} s| p>=110 \pm 60 \mathrm{MeV}, \quad y=0.20 \pm 0.12, \quad \hat{\sigma}=35 \pm 6 \mathrm{MeV} \quad .
$$

Note the large value of the matrix element $m_{s}<p|\bar{s} s| p>=-671 \mathrm{MeV}$ in dimensional regularization. This time the chiral expansions of the $\sigma$-terms in the cutoff scheme for $\Lambda=400 \mathrm{MeV}$ are

$$
\begin{aligned}
& \sigma_{\pi N}(0)=31.2+13.8 \mathrm{MeV}=45.0 \mathrm{MeV} \\
& \sigma_{K N}^{(1)}(0)=289.7+100.4 \mathrm{MeV}=390.1 \mathrm{MeV} \\
& \sigma_{K N}^{(2)}(0)=173.9+83.4 \mathrm{MeV}=257.3 \mathrm{MeV}
\end{aligned}
$$

whereas the chiral expansions of the $\sigma$-terms in dimensional regularization read

$$
\begin{aligned}
& \sigma_{\pi N}(0)=140.7-95.7 \mathrm{MeV}=45.0 \mathrm{MeV} \\
& \sigma_{K N}^{(1)}(0)=1060-1106 \mathrm{MeV}=-46 \mathrm{MeV} \\
& \sigma_{K N}^{(2)}(0)=557-658 \mathrm{MeV}=-101 \mathrm{MeV} .
\end{aligned}
$$

As in the case without resonances the convergence of the chiral series in the cutoff scheme is significantly improved with respect to dimensional regularization. We do not observe a dramatic change in the results as suggested by employing dimensional regularization. For the evaluation of the shifts of the scalar formfactors to the Cheng-Dashen points we applied the regularization scheme $R_{2}$ since the scheme $R_{1}$ leads to divergent integrals. For the $\sigma_{\pi N}$ shift we find

$$
\sigma_{\pi N}\left(2 m_{\pi}^{2}\right)-\sigma_{\pi N}(0)=6 \pm 1 \mathrm{MeV}
$$

There is still a sizeable uncertainty in the $K N \sigma$-terms, and we present only the results for $\Lambda=400$ and $500 \mathrm{MeV}$ in Table 4 . 


\section{Summary}

In this paper, we have evaluated the $\pi N$ and $K N \sigma$-terms and scalar form factors by using a cutoff regularization.

o First, we calculated the $\sigma$-terms by using the next-to-leading order Lagrangian for the Goldstone bosons and the lowest-lying baryon octet in the heavy baryon formulation. The Goldstone boson integrals are evaluated by using a simple dipole regulator with a cutoff $\Lambda$ proposed in [3]. We also used a modified regulator which is similar to the first one for vanishing off-shell momenta of the external baryons. We have given the complete expressions for the $\sigma$-terms up to the order $q^{3}$, where $q$ is an external momentum or meson mass. The cutoff parameter induces an additional mass scale that does not vanish in the chiral limit and, therefore, destroys the strict chiral counting scheme. We are able to show that to the order we are working the physics does not depend on $\Lambda$, since one is able to absorb the effects of $\Lambda$ into a renormalization of the coupling constants.

- The spin-3/2 decuplet is separated from the octet by $231 \mathrm{MeV}$ in average which is smaller than the kaon or eta mass. Therefore, we proceeded by adding the decuplet to the effective theory. Performing the calculation with the regulator from [3] leads to divergent integrals for non-vanishing off-shell momenta. One obtains finite results for the other regulator.

- There are four unknown parameters in the theory - the coupling constants $b_{0, D, F}$ from the Lagrangian of chiral order $q^{2}$ and the baryon mass in the chiral limit $\stackrel{\circ}{M}$ - which have to be fixed from phenomenology. We choose the four baryon masses in the isospin limit $(N, \Sigma, \Lambda, \Xi)$ and the value $\sigma_{\pi N}(0)=45 \mathrm{MeV}$ to perform a least-squares fit for these parameters. In our analysis the cutoff parameter ranges from 300 to $600 \mathrm{MeV}$ to account for all the long distance physics, but little of the short distance physics, which are not described appropriately by the effective theory, is included. Predictions for the $K N \sigma^{-}$ terms and the strange contribution to the nucleon mass are made. The results without the decuplet are $\sigma_{K N}^{(1)}(0)=400 \pm 30 \mathrm{MeV}$ and $\sigma_{K N}^{(2)}(0)=270 \pm 20 \mathrm{MeV}$ for the two $K N$ $\sigma$-terms (accounting for the uncertainty in $\Lambda$ ). The strange contribution to the nucleon mass is $\left\langle p\left|m_{s} \bar{s} s\right| p>=150 \pm 50 \mathrm{MeV}\right.$ which translates into the strangeness fraction $y=$ $0.25 \pm 0.05$ and $\hat{\sigma}=33 \pm 3 \mathrm{MeV}$. The results are in good agreement with previous calculations [6, 7]. While a definite statement about the convergence of the chiral expansions for the $\sigma$-terms cannot be made in dimensional regularization, there is a clear convergence in the cutoff scheme. The $\pi N \sigma$-term shift to the Cheng-Dahen point is $4 \pm 1 \mathrm{MeV}$ in both cutoff schemes. This number is in agreement with the complete fourth order calculation in conventional heavy baryon chiral perturbation theory. But this value is smaller than the dispersive calculation of [6]. It remains to be seen how higher order corrections not yet accounted for will modify this result. The shifts for the $K N \sigma$-terms depend strongly on the value of $\Lambda$ which might indicate that higher chiral orders are important. In order to include the decuplet states into the fit, one has to account for the decuplet contributions to the baryon masses. Again a least-squares fit is performed and it turns out that there are no significant changes in the results. One obtains $\sigma_{K N}^{(1)}(0)=380 \pm 40 \mathrm{MeV}, \sigma_{K N}^{(2)}(0)=250 \pm 30$ $\mathrm{MeV},<p\left|m_{s} \bar{s} s\right| p>=110 \pm 60 \mathrm{MeV}, y=0.20 \pm 0.12$ and $\hat{\sigma}=35 \pm 6 \mathrm{MeV}$. For the $\pi N$ $\sigma$-term shift we obtain $6 \pm 1 \mathrm{MeV}$. 


\section{Acknowledgements}

I am grateful to John Donoghue for useful discussions and reading the manuscript. This work was supported in part by the Deutsche Forschungsgemeinschaft.

\section{A Decuplet contributions to the scalar formfactors}

The decuplet contributions to the scalar formfactors for non-vanishing momentum transfer $t$ can be presented as follows

$$
\begin{aligned}
\delta \sigma_{\pi N}(t) & =-\frac{m_{\pi}^{2} C^{2}}{96 \pi F_{\pi}^{2}} \Lambda^{4}\left(8 \tilde{H}\left(m_{\pi}\right)+\tilde{H}\left(m_{K}\right)\right) \\
\delta \sigma_{K N}^{(1)}(t) & =-\frac{m_{K}^{2} C^{2}}{96 \pi F_{\pi}^{2}} \Lambda^{4}\left(4 \tilde{H}\left(m_{\pi}\right)+\frac{4}{3} \tilde{H}\left(m_{K}\right)\right) \\
\delta \sigma_{K N}^{(2)}(t) & =-\frac{m_{K}^{2} C^{2}}{96 \pi F_{\pi}^{2}} \Lambda^{4}\left(4 \tilde{H}\left(m_{\pi}\right)+2 \tilde{H}\left(m_{K}\right)\right)
\end{aligned}
$$

with

$$
\begin{aligned}
\tilde{H}\left(m_{\phi}\right)= & \frac{1}{\left(\Lambda^{2}-m_{\phi}^{2}\right)^{2}}\left(-2 \Delta\left[\sqrt{\frac{m_{\phi}^{2}}{t}-\frac{1}{4}} \arcsin \frac{\sqrt{t}}{2 m_{\phi}}+\sqrt{\frac{\Lambda^{2}}{t}-\frac{1}{4}} \arcsin \frac{\sqrt{t}}{2 \Lambda}\right]\right. \\
& +\Delta\left[\frac{m_{\phi}^{2}-\Lambda^{2}}{2 t} \ln \frac{m_{\phi}^{2}}{\Lambda^{2}}+\frac{1}{t} \beta\left(\operatorname{artanh} \frac{m_{\phi}^{2}-\Lambda^{2}+t}{\beta}-\operatorname{artanh} \frac{m_{\phi}^{2}-\Lambda^{2}-t}{\beta}\right)\right] \\
& -\left[\Delta^{2}-\Lambda^{2}+\frac{1}{2} t\right] \int_{0}^{1} d x \frac{1}{\sqrt{\Lambda^{2}-\Delta^{2}-x(1-x) t}} \arccos \frac{\Delta}{\sqrt{\Lambda^{2}-x(1-x) t}} \\
& +\left[2 \Delta^{2}-m_{\phi}^{2}-\Lambda^{2}+t\right] \int_{0}^{1} d x \frac{1}{\sqrt{\Lambda^{2}-x\left[\Lambda^{2}-m_{\phi}^{2}\right]-\Delta^{2}-x(1-x) t}} \\
& \times \arccos \frac{\Delta}{\left.\sqrt{\Lambda^{2}-x\left[\Lambda^{2}-m_{\phi}^{2}\right]-x(1-x) t}-\left[\Delta^{2}-m_{\phi}^{2}+\frac{1}{2} t\right] f\left(m_{\phi}\right)\right)}
\end{aligned}
$$

with

$$
\beta=\sqrt{\left(m_{\phi}^{2}-\Lambda^{2}-t\right)^{2}-4 t \Lambda^{2}}
$$

and

$$
\begin{aligned}
& f\left(m_{\phi}\right)=\int_{0}^{1} d x \frac{1}{\sqrt{\Delta^{2}-m_{\phi}^{2}+x(1-x) t}} \ln \frac{\Delta+\sqrt{\Delta^{2}-m_{\phi}^{2}+x(1-x) t}}{\sqrt{m_{\phi}^{2}-x(1-x) t}} ; \text { for } m_{\phi}<\Delta \\
& f\left(m_{\phi}\right)=\int_{0}^{1} d x \frac{1}{\sqrt{m_{\phi}^{2}-\Delta^{2}-x(1-x) t}} \arccos \frac{\Delta}{\sqrt{m_{\phi}^{2}-x(1-x) t}} ; \text { for } m_{\phi}>\Delta
\end{aligned}
$$

where we required $\Lambda>\Delta$. We presented the result for sufficiently small $t$. With increasing $t$ the squareroots become imaginary and one has to continue $\tilde{H}\left(m_{\phi}\right)$ analytically. 


\section{B Decuplet contributions to the masses}

In this Appendix we give the results for the decuplet contributions to the masses. They can be written in the form

$$
\delta M_{B}=\frac{C^{2}}{24 \pi F_{\pi}^{2}} \Lambda^{4}\left(\alpha_{B}^{\pi} M\left(m_{\pi}\right)+\alpha_{B}^{K} M\left(m_{K}\right)+\alpha_{B}^{\eta} M\left(m_{\eta}\right)\right)
$$

with coefficients

$$
\begin{array}{lll}
\alpha_{N}^{\pi}=4, & \alpha_{N}^{K}=1, & \alpha_{N}^{\eta}=0 ;
\end{array}
$$

and

$$
\begin{aligned}
M\left(m_{\phi}\right)=\frac{1}{\left(\Lambda^{2}-m_{\phi}^{2}\right)^{2}} & \left(\frac{1}{2} \Delta\left[\frac{3}{2} m_{\phi}^{2}-\Delta^{2}\right] \ln \frac{m_{\phi}^{2}}{\Lambda^{2}}-\frac{1}{4} \Delta\left[m_{\phi}^{2}-\Lambda^{2}\right]\right. \\
- & {\left[\Delta^{2}-m_{\phi}^{2}\right]^{3 / 2} \ln \left[\frac{\Delta}{m_{\phi}}+\sqrt{\left.\frac{\Delta^{2}}{m_{\phi}^{2}}-1\right]}\right]+\left[\Lambda^{2}-\Delta^{2}\right]^{3 / 2} \arccos \frac{\Delta}{\Lambda} } \\
+ & \left.\frac{3}{2}\left[m_{\phi}^{2}-\Lambda^{2}\right]\left[\Lambda^{2}-\Delta^{2}\right]^{1 / 2} \arccos \frac{\Delta}{\Lambda}\right) ; \quad \text { for } m_{\phi}<\Delta \\
M\left(m_{\phi}\right)=\frac{1}{\left(\Lambda^{2}-m_{\phi}^{2}\right)^{2}}\left(\frac{1}{2} \Delta\left[\frac{3}{2} m_{\phi}^{2}-\Delta^{2}\right] \ln \frac{m_{\phi}^{2}}{\Lambda^{2}}-\frac{1}{4} \Delta\left[m_{\phi}^{2}-\Lambda^{2}\right]\right. & \quad\left[m_{\phi}^{2}-\Delta^{2}\right]^{3 / 2} \arccos \frac{\Delta}{m_{\phi}}+\left[\Lambda^{2}-\Delta^{2}\right]^{3 / 2} \arccos \frac{\Delta}{\Lambda} \\
+ & \left.\frac{3}{2}\left[m_{\phi}^{2}-\Lambda^{2}\right]\left[\Lambda^{2}-\Delta^{2}\right]^{1 / 2} \arccos \frac{\Delta}{\Lambda}\right) ; \quad \text { for } m_{\phi}>\Delta
\end{aligned}
$$

where we required $\Lambda>\Delta$.

\section{References}

[1] J. Gasser, H. Leutwyler, Ann. Phys. (NY) 158 (1984), 142; Nucl. Phys. B250 (1985), 465

[2] J.F. Donoghue, B.R. Holstein, hep-ph/9803312, Phys. Lett. B ( in press )

[3] J.F. Donoghue, B.R. Holstein, B. Borasoy, UMass preprint (1998), hep-ph/9804281

[4] E. Jenkins, A.V. Manohar, Phys. Lett. B259 (1991), 353

[5] V. Bernard, N. Kaiser, U.-G. Meißner, Z. Phys. C60 (1993), 111

[6] J. Gasser, H. Leutwyler, M.E. Sainio, Phys. Lett. B253 (1991), 252, 260

[7] J. Gasser, Ann. Phys. 136 (1981), 62

[8] B. Borasoy, Ph.D. Thesis, University of Bonn, 1996

[9] E. Jenkins, A.V. Manohar, in: Effective field theories of the standard model, U.-G. Meißner (ed.). Singapore: World Scientific 1992 


\section{Table captions}

Table 1 Given are the LECs $b_{0, D, F}$, the renormalized baryon mass in the chiral limit $\stackrel{\circ}{M}_{r}$, the two $K N \sigma$-terms, the strange quark matrix element (SME), the strange quark fraction $y, \hat{\sigma}$ and the deviation from the Gell-Mann-Okubo relation $\left[3 M_{\Lambda}+M_{\Sigma}-2 M_{N}-2 M_{\Xi}\right] / 4$ in dimensional regularization and for various values of the cutoff parameter $\Lambda$ in $\mathrm{MeV}$.

Table 2 Shifts to the Cheng-Dashen points in $\mathrm{MeV}$ in dimensional regularization and for both regularization schemes $R_{1}$ and $R_{2}$. The cutoff parameter $\Lambda$ is given in MeV.

Table 3 Results of the calculation including the spin-3/2 decuplet. The cutoff parameter $\Lambda$ is given in $\mathrm{MeV}$.

Table 4 Shifts to the Cheng-Dashen points in $\mathrm{MeV}$ in dimensional regularization and for the regularization scheme $R_{2}$ and including the decuplet. The cutoff parameter $\Lambda$ is given in $\mathrm{MeV}$. 


\begin{tabular}{|c|c|c|c|c|c|}
\hline & dim. & $\Lambda=300$ & $\Lambda=400$ & $\Lambda=500$ & $\Lambda=600$ \\
\hline $\begin{array}{ll}b_{D} & {\left[\mathrm{GeV}^{-1}\right]}\end{array}$ & 0.008 & 0.068 & 0.070 & 0.072 & 0.075 \\
\hline$b_{F} \quad\left[\mathrm{GeV}^{-1}\right]$ & -0.606 & -0.197 & -0.185 & -0.171 & -0.153 \\
\hline$b_{0} \quad\left[\mathrm{GeV}^{-1}\right]$ & -0.786 & -0.459 & -0.417 & -0.371 & -0.320 \\
\hline$\stackrel{\circ}{M}_{r}[\mathrm{GeV}]$ & 1.011 & 0.701 & 0.720 & 0.738 & 0.755 \\
\hline$\sigma_{K N}^{(1)}(0) \quad[\mathrm{MeV}]$ & 157.4 & 420.9 & 407.1 & 393.5 & 380.6 \\
\hline$\sigma_{K N}^{(2)}(0) \quad[\mathrm{MeV}]$ & 120.4 & 285.5 & 275.0 & 265.0 & 255.8 \\
\hline SME $\quad[\mathrm{MeV}]$ & -271.6 & 187.5 & 162.6 & 138.2 & 115.2 \\
\hline$y$ & -0.488 & 0.337 & 0.292 & 0.248 & 0.207 \\
\hline$[\mathrm{MeV}]$ & 67.0 & 29.8 & 31.8 & 33.8 & 35.7 \\
\hline$[\mathrm{MeV}]$ & 4.4 & 0.2 & 0.3 & 0.4 & 0.6 \\
\hline
\end{tabular}

Table 1

\begin{tabular}{l|c|c|c} 
& $\sigma_{\pi N}\left(2 m_{\pi}^{2}\right)-\sigma_{\pi N}(0)$ & $\sigma_{K N}^{(1)}\left(2 m_{K}^{2}\right)-\sigma_{K N}^{(1)}(0)$ & $\sigma_{K N}^{(2)}\left(2 m_{K}^{2}\right)-\sigma_{K N}^{(2)}(0)$ \\
\hline $\operatorname{dim}$. & 7.4 & $318.9+i 334.9$ & $9.4+i 334.9$ \\
\hline$\Lambda=300\left(R_{1}\right)$ & 3.2 & $2.3-i 1.1$ & $-73.1+i 3.4$ \\
$\Lambda=400\left(R_{1}\right)$ & 3.8 & $-22.8-i 7.4$ & $-107.5+i 22.2$ \\
$\Lambda=500\left(R_{1}\right)$ & 4.3 & $7.6-i 94.0$ & $-199.3+i 281.9$ \\
$\Lambda=600\left(R_{1}\right)$ & 4.6 & $-220.1+i 373.4$ & $-1564+i 373.4$ \\
\hline$\Lambda=300\left(R_{2}\right)$ & 3.6 & $-35.2+i 65.7$ & $35.7-i 1.9$ \\
$\Lambda=400\left(R_{2}\right)$ & 4.1 & $-84.8-i 127.6$ & $-45.5-i 266.7$ \\
$\Lambda=500\left(R_{2}\right)$ & 4.5 & $-347.9+i 92.6$ & $-220.9-i 243.3$ \\
$\Lambda=600\left(R_{2}\right)$ & 4.9 & $-123.6+i 373.4$ & $-669.8+i 373.4$ \\
\hline
\end{tabular}

Table 2 


\begin{tabular}{|c|c|c|c|c|c|}
\hline & dim. & $\Lambda=300$ & $\Lambda=400$ & $\Lambda=500$ & $\Lambda=600$ \\
\hline $\begin{array}{ll}b_{D} & {\left[\mathrm{GeV}^{-1}\right]}\end{array}$ & 0.510 & 0.061 & 0.055 & 0.046 & 0.035 \\
\hline$b_{F} \quad\left[\mathrm{GeV}^{-1}\right]$ & -1.022 & -0.191 & -0.173 & -0.149 & -0.120 \\
\hline$b_{0} \quad\left[\mathrm{GeV}^{-1}\right]$ & -1.591 & -0.424 & -0.351 & -0.264 & -0.169 \\
\hline$\stackrel{\circ}{M}_{r}[\mathrm{GeV}]$ & 1.328 & 0.730 & 0.761 & 0.787 & 0.806 \\
\hline$\sigma_{K N}^{(1)}(0) \quad[\mathrm{MeV}]$ & -46.0 & 411.0 & 390.2 & 369.1 & 348.7 \\
\hline$\sigma_{K N}^{(2)}(0) \quad[\mathrm{MeV}]$ & -100.7 & 275.2 & 257.3 & 239.3 & 222.2 \\
\hline SME $\quad[\mathrm{MeV}]$ & -671.3 & 168.3 & 129.7 & 90.7 & 53.1 \\
\hline$y$ & -1.206 & 0.302 & 0.233 & 0.163 & 0.095 \\
\hline$[\mathrm{MeV}]$ & 99.3 & 29.8 & 31.4 & 34.5 & 40.7 \\
\hline$[\mathrm{MeV}]$ & 11.0 & 0.3 & 0.6 & 0.9 & 1.2 \\
\hline
\end{tabular}

Table 3

\begin{tabular}{c|c|c|c} 
& $\sigma_{\pi N}\left(2 m_{\pi}^{2}\right)-\sigma_{\pi N}(0)$ & $\sigma_{K N}^{(1)}\left(2 m_{K}^{2}\right)-\sigma_{K N}^{(1)}(0)$ & $\sigma_{K N}^{(2)}\left(2 m_{K}^{2}\right)-\sigma_{K N}^{(2)}(0)$ \\
\hline$\Lambda=400\left(R_{2}\right)$ & 5.7 & $-292.3+i 73.4$ & $-228.1-i 65.8$ \\
$\Lambda=500\left(R_{2}\right)$ & 6.5 & $-534.8+i 425.7$ & $-401.6+i 89.8$ \\
\hline
\end{tabular}

Table 4 